\title{
A flexible and efficient multi-model framework in support of water management
}

\author{
Vincent Wolfs ${ }^{1}$, Quan Tran Quoc ${ }^{1}$, and Patrick Willems ${ }^{1,2}$ \\ ${ }^{1}$ KU Leuven, Hydraulics Division, Kasteelpark Arenberg 40 box 2448, 3001 Leuven, Belgium \\ ${ }^{2}$ Vrije Universiteit Brussel, Department of Hydrology and Hydraulic Engineering, Brussels, Belgium
}

Correspondence to: Vincent Wolfs (vincent.wolfs@bwk.kuleuven.be)

Published: 12 May 2016

\begin{abstract}
Flexible, fast and accurate water quantity models are essential tools in support of water management. Adjustable levels of model detail and the ability to handle varying spatial and temporal resolutions are requisite model characteristics to ensure that such models can be employed efficiently in various applications. This paper uses a newly developed flexible modelling framework that aims to generate such models. The framework incorporates several approaches to model catchment hydrology, rivers and floodplains, and the urban drainage system by lumping processes on different levels. To illustrate this framework, a case study of integrated hydrologicalhydraulic modelling is elaborated for the Grote Nete catchment in Belgium. Three conceptual rainfall-runoff models (NAM, PDM and VHM) were implemented in a generalized model structure, allowing flexibility in the spatial resolution by means of an innovative disaggregation/aggregation procedure. They were linked to conceptual hydraulic models of the rivers in the catchment, which were developed by means of an advanced model structure identification and calibration procedure. The conceptual models manage to emulate the simulation results of a detailed full hydrodynamic model accurately. The models configured using the approaches of this framework are well-suited for many applications in water management due to their very short calculation time, interfacing possibilities and adjustable level of detail.
\end{abstract}

\section{Introduction}

Water management is constantly evolving: there is a shift from more traditional end-of-pipe solutions to a holistic and source-oriented approach, new technologies emerge and multiple trends have a major impact on the water system, such as climate change and the increasing urbanization. Integrated analyses are needed that require broadening the investigated scale and scope to identify cost-effective and futureproof solutions.

To cope with these changes and to ensure that such strategies can be identified, flexible modelling frameworks are needed that allow the water manager to easily and efficiently combine available data bases, real-time measurements and the most suitable models for every component of the holistic water system. The water system is characterized by multiple processes, each acting on different spatial and temporal scales. For instance, CSOs can spill into rivers during a short time frame, but their pollution can disperse and deteriorate the river's water quality for a much longer period. Therefore, the efficient use of spatially distributed data sets, and models with different degrees of model detail and varying spatial and temporal resolutions play a pivotal role in water management analyses. Within this context, we developed a flexible and computationally efficient modelling methodology to simulate the catchment hydrology and the hydraulics of rivers and urban drainage systems. The model detail can easily be adjusted, enabling the modeller to focus on the dominating processes at various scales.

Section 2 discusses this hydrological-hydraulic modelling framework. The approach is demonstrated for the Grote Nete catchment in Belgium. A flexible hydrological model was set up to simulate the catchment rainfall runoff at different spatial resolutions, and a conceptual hydraulic model was created of all main rivers in the catchment. Section 3 describes the study area. Section 4 shows the main results. Finally, the 
potential of the developed approach is discussed and a view on future developments is given.

\section{Methodology}

Figure 1 schematizes the developed flexible modelling framework to parsimoniously model the water system at the catchment scale. The modelling framework includes tailored approaches to model the hydrological and hydraulic (both the river and floodplains, and the sewer system) components of the water system. The following paragraphs elaborate on the developed hydrological and hydraulic modelling approaches.

\subsection{Hydrological modelling approach}

For catchment rainfall-runoff modelling, numerous different hydrological model conceptualizations and parameterizations exist. In order to allow flexibility in the spatial resolution, while maintaining consistency in results when spatial resolution is changed, and to allow flexible changes in process equations, we developed a flexible hydrological modelling approach (Tran Quoc and Willems, 2016). This methodology reasons from a generalized lumped model structure that can be disaggregated at various scales to add spatial detail. The use of such an approach that requires only a few parameters to be calibrated is frequently suggested in literature to obviate equifinality issues encountered in hydrological modelling (e.g. Jakeman et al., 1990; Wheather et al., 1993). Also, their parsimonious configuration not only facilitates and speeds up model calibration, but their performance is on average higher compared to more complex distributed models (e.g. Ajami et al., 2004; Breuer et al., 2009). The entire modelling framework is implemented in PCRaster - Python. Currently, three conceptual rainfall-runoff models (PDM, Moore, 2007; NAM, Nielsen and Hansen, 1973; and VHM, Willems, 2014) are incorporated because of their widespread use in Belgium. The concept of a flexible model structure also allows the use of an ensemble approach involving multiple model types to simulate the rainfall-runoff, thereby obviating the election of one model type over others.

Our modelling framework allows convenient, semiautomated disaggregation of the lumped model components to higher spatial resolutions. This allows tailoring model detail to the characteristics of specific areas or to the needs for intended applications. This also broadens the range of possible applications, including spatial scenario simulations. The disaggregation is achieved by mapping the different model components on a grid with varying cell sizes. To ensure that consistent results are obtained and the parameters have smooth transitions with varying spatial scales, the lumped parameters are disaggregated based on spatial catchment characteristics, such as the topography, land use and soil parameters. Only few additional calibration parameters are required to scale the relative spatial differences in model parameters. In the opposite direction, aggregation allows to

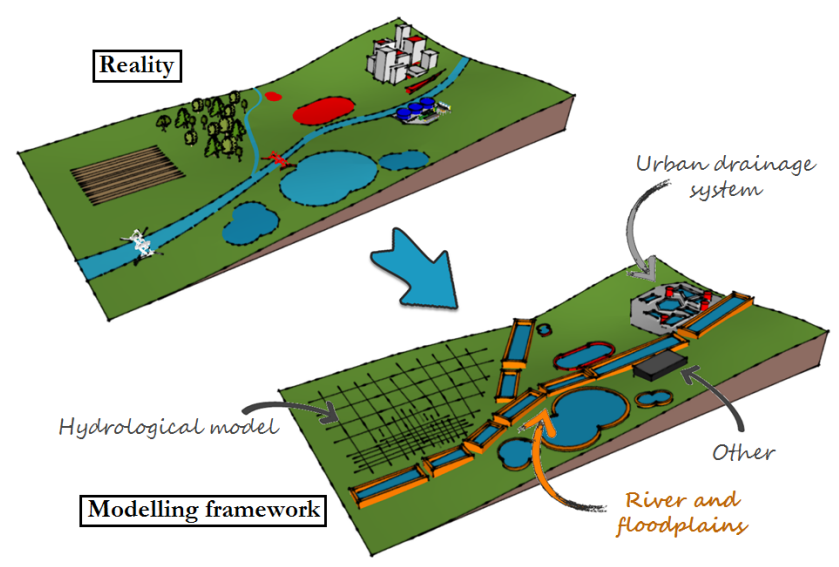

Figure 1. Schematization of the proposed modelling framework.

spatially lump processes and transfer a highly spatially detailed model to a more lumped model. For further details on this approach, the reader is referred to Tran Quoc and Willems (2016).

\subsection{Hydraulic modelling approach}

Hydrodynamic models, which are based on the de SaintVenant equations, have become the standard tool for water practitioners to simulate rivers and sewer systems. Such models are generally very accurate, but the level of model detail cannot be adjusted, leading to overly complex models for many applications and analyses. Their complicatedness leads to prolonged calculation times. In addition, it is very often difficult or even impossible to interface different models. Linking such models would in turn lead to an even increased complicatedness.

Therefore, we developed a new flexible conceptual modelling approach for rivers and sewer systems. The modelling approach simplifies the network topology and momentum equations of the detailed hydrodynamic models according to a lower-fidelity approach (Ravazi et al., 2012) to strike the balance between model accuracy, and computational efficiency and model detail. The modelling approach is incorporated in the CMD software.

The modelling approach reasons from the storage cell concept, in which the entire system is divided into multiple interconnected reservoirs. In each such reservoir, the continuity equation is applied to close the water balance. The delineation of rivers and sewer systems into reservoirs is very flexible: additional reservoirs and model detail can be added in areas where enhanced spatial detail is necessary, while processes can be lumped on larger scales at other locations. A broad range of conventionally applied model structures, such as the linear reservoir theory and transfer functions, were combined with advanced machine learning techniques in a mechanistic and modular framework to fill in the momentum equations. This modularity allows the modeller to pick the 
most appropriate model structure based on the dynamics of the river or sewer system, the desired accuracy, available data and the intended applications. Due to variety of model structures, more complex dynamics can also be emulated accurately on various scales, such as backwater effects, and pressurized and reverse flows in pipes. Details of this modelling approach and software can be found in Wolfs et al. (2015).

This conceptual modelling approach has been used before for various applications and case studies, including flood probability mapping (Wolfs et al., 2012), real-time control of hydraulic structures to prevent flooding (Vermuyten et al., 2015), an impact analysis of CSOs on river water quality (Keupers et al., 2015) and the quantification of source control versus end-of-pipe solutions on flood probabilities in a coupled river-sewer system (De Vleeschauwer et al., 2014). In these studies, the conceptual models were calibrated to simulation results of detailed hydrodynamic models.

\subsection{Model interfacing}

Interfacing between hydrological and hydraulic models may be bidirectional, but unidirectional coupling was considered so far in this study, where results from the hydrological model are transferred to the hydraulic model at its spatial resolution. The hydrological model is coded in PCRasterPython, while the hydraulic model is implemented in a $\mathrm{C}++$ executable that can run on Windows and Unix systems. The results of the hydrological model are stored in ASCII format, those of the hydraulic model in netCDF. A simple routine handles the requisite data processing steps when transferring data from the hydrological to the hydraulic model. These include the transformation of data formats, and the adjustment of the save interval (spatial and temporal) of all time series, since the hydraulic model itself does not alter the save interval of its input time series. This routine also enables the hydrological and hydraulic models to run in different software environments, thereby obviating model code adaptations. The data transferring routine was coded in MATLAB, since this environment can easily and efficiently handle the used data standards of the hydrological and hydraulic models. Interfacing was done manually for this study, but this process is currently being formalized and automated by the authors. A web processing service interfacing is also being set up to enable interfacing with models and databases in a network.

\section{Case study}

The hydrological and hydraulic modelling approaches were tested on the Grote Nete catchment in Belgium (see Fig. 2). The catchment has a total area of $385 \mathrm{~km}^{2}$. The most dominant land use types in the watershed are cropland (33\%), forest (23\%), grassland (19\%) and built-up area (19\%). The region has predominant sandy soil coverage with parabolic land dunes in the upstream parts, while the downstream

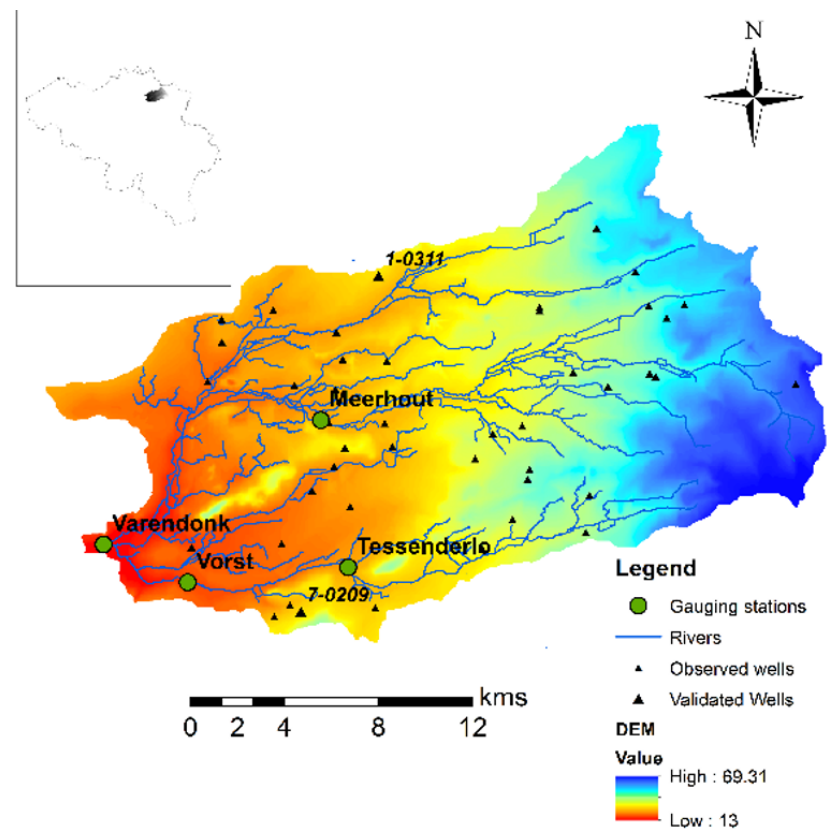

Figure 2. The Grote Nete catchment with the internal flow stations at Meerhout, Vorst and Tessenderlo, and the outlet station at Varendonk.

parts are characterized by wide alluvial planes with shallow groundwater. The catchment contains numerous river tributaries, and a dense network of ditches and subsurface drains that feed into the larger Grote Nete, Molse Nete and Grote Laak rivers.

Four flow gauging stations, six rain gauges and 30 observation wells which monitor groundwater heads are present in the catchment (see Fig. 2). In an earlier study (Vansteenkiste et al., 2014), NAM, PDM and VHM lumped conceptual models were calibrated for this region using a step-wise approach based on the rainfall and flow measurements. The reader is referred to that study for details on this calibration procedure. In addition, a detailed hydrodynamic MIKE11 model was configured that comprises these rivers and their main tributaries. This MIKE11 model uses the simulation results of the VHM model as rainfall-runoff input.

First, the generalized structure of the hydrological models was set-up by implementing the parameters of the earlier calibrated NAM, PDM and VHM models. Next, the parameters were spatially disaggregated using land use and soil texture maps with a $250 \mathrm{~m}$ resolution, leading to six hydrological models in total (NAM, PDM and VHM implementation; each lumped and spatially disaggregated). Two adjustment factors were calibrated for the spatial disaggregation to compensate for the changes in subflow distribution by the grid-togrid routing compared with the lumped model. In addition, two parameters were calibrated to account for spatial variations in catchment characteristics. The hydrological models use hourly catchment averaged rainfall after applying the 

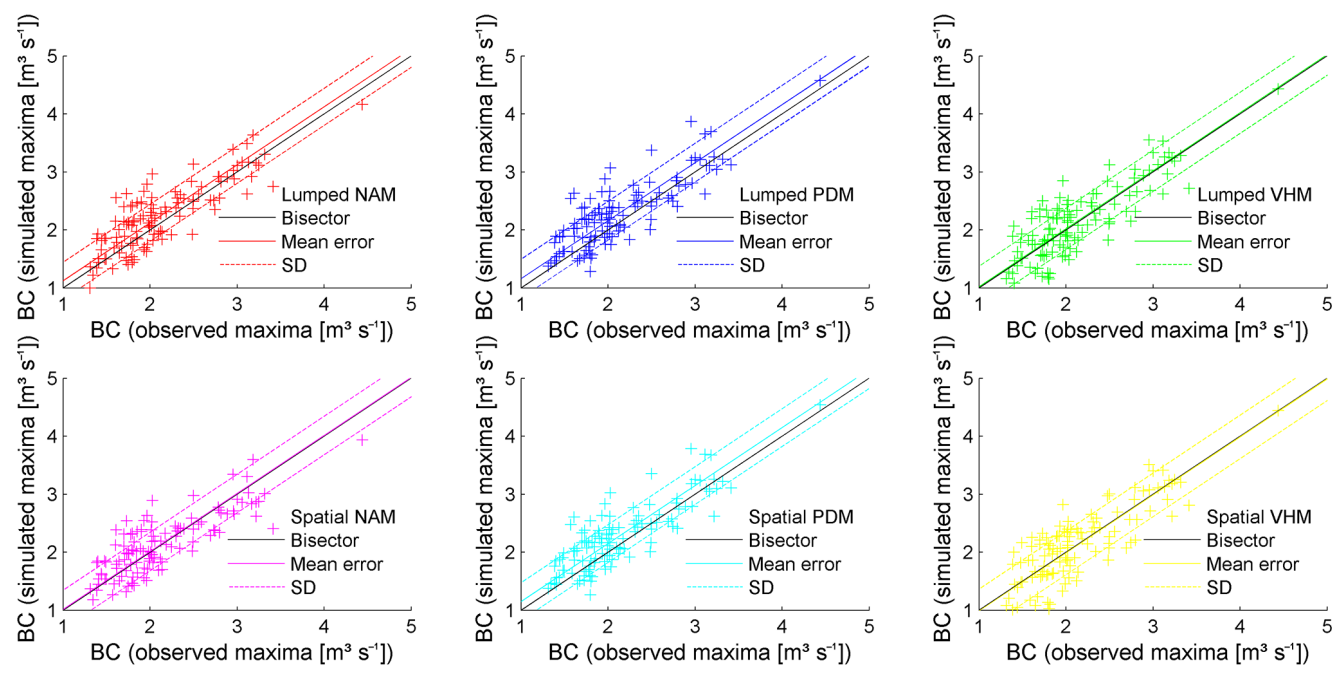

Figure 3. Simulated versus observed peak flows after Box-Cox transformation $(\lambda=0.25)$, for the spatial and lumped models at the Grote Nete downstream catchment station, for the full simulation period 13 August 2002-31 December 2008.
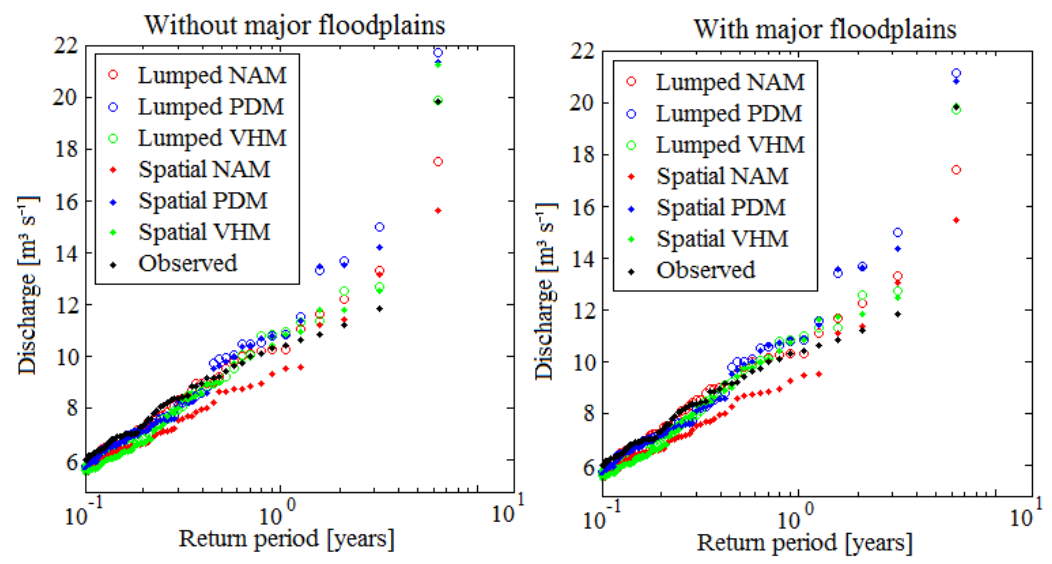

Figure 4. Simulated versus observed peak flow frequency distributions, for the spatial and lumped models at the Grote Nete downstream catchment station, for the full simulation period 13 August 2002-31 December 2008, with and without the effect of the three floodplains with highest capacity.

Thiessen polygon method to the six available rain gauges. The period of 13 May 2002-31 December 2005 was used for calibration, and 1 January 2006-31 December 2008 for validation.

The conceptual hydraulic model was configured for the main rivers in the Grote Nete catchment (see also Fig. 2). Given the availability of an existing and validated detailed hydrodynamic MIKE11 model, and due to the lack of enough measurement data, simulation results of the detailed model were used to calibrate and validate the conceptual model. Calibration was performed on the period 8 March 200111 April 2001, while validation was done for 12 April 200131 December 2008. The investigated river reaches were divided in 7 reservoirs. The boundaries of most reservoirs mostly coincide with bridges or culverts. Note that it is easily possible to adjust the number of calculation nodes, and thus to include additional model detail in specific areas. The main floodplains with a total capacity of $200000 \mathrm{~m}^{3}$ were also incorporated in the conceptual model. These floodplains are situated just upstream of the outlet station of the catchment. After the model topology was defined, the most appropriate model structures were identified and calibrated. The flow was predominantly modelled using transfer functions, while rating curves were applied to model the water levels. Besides visual comparison of the conceptual and detailed hydrodynamic model results, the NSE values were also calculated using the conceptual and MIKE11 simulation results. The performance of the hydraulic model is exceptionally good, with NSE values for all flows and water level results exceeding 0.985 for both the calibration and validation events.

Each of the six conceptual rainfall-runoff models (NAM, PDM, VHM; and for both the lumped and spatially dis- 
tributed versions) were interfaced with the conceptual river model. In this way, six different integrated models were obtained.

\section{Results}

The results of the interfaced hydrological - hydraulic conceptual models were evaluated based on the flow observed at the catchment downstream gauging station. This is done using four methods: (i) visual comparison of the observed and simulated river flows at the gauging stations, (ii) evaluation of a set of five statistical measures, (iii) control of the water balance and (iv) analysis of evaluation plots focusing on extremes and cumulative values as proposed in Willems (2009).

For the peak flow extremes (Figs. 3, 4), the spatial and lumped models show similar performance and frequency distributions: whereas the peak flow error (PFE) after BoxCox (BC) transformation (Box and Cox, 1964) ranges between -0.10 and $0.15 \mathrm{BC}\left(\mathrm{m}^{3} \mathrm{~s}^{-1}\right)(\mathrm{BC}$ parameter $\lambda=0.25)$ for the lumped models, its value range between 0.11 and $0.18 \mathrm{BC}\left(\mathrm{m}^{3} \mathrm{~s}^{-1}\right)$ for the spatial models. For PDM, the spatial models are slightly more accurate or of equal accuracy for the peak flows as the lumped models, whereas for other models (NAM, VHM) the opposite is observed. The differences are, however, for all models minor.

Figure 4 shows the empirical peak flow frequency distribution for all configured models at the most downstream station. In order to investigate the influence of the river flooding on the river peak flow dynamics and statistics, the hydraulic model simulations were conducted with and without the floodplains with the highest capacity. It is seen in Fig. 4 that the floodplains only affect the highest flows. Closer inspection of the simulation results revealed that only two of the three floodplains were filled during the entire simulation period of more than six years, explaining the minor influence on the peak flows for the lower return periods. Note that all models overestimate the flow for empirical return periods between one and three years. Including also the numerous much smaller floodplains along the more upstream tributaries in the catchment will likely reduce the simulated flows in that range of return periods.

A similar conclusion holds for the low flow extremes. The low flow accuracy is fair for both the lumped and spatial models: LFE between 0.02 and $0.18 \mathrm{BC}\left(\mathrm{m}^{3} \mathrm{~s}^{-1}\right)(\lambda=0.25)$. One again does not see strong differences in the low flow performance between the lumped models (LFE between 0.04 and $0.18 \mathrm{BC}\left(\mathrm{m}^{3} \mathrm{~s}^{-1}\right)$ ) and the spatial models (LFE between 0.02 and $0.18 \mathrm{BC}\left(\mathrm{m}^{3} \mathrm{~s}^{-1}\right)$ ). The cumulative runoff volumes are for many models slightly underestimated but limited to $10.63 \%$, which is fair. Most models show lower differences: WBE between -10.63 and $+4.11 \%$ for the spatial models and between -10.11 and $-0.80 \%$ for the lumped models.

Given this good accuracy, the models hence can be considered useful for impact studies on peak flow extremes (flood studies), low flow extremes (water availability studies) and cumulative runoff volumes (water balance studies). The spatial models are applicable for scenario studies that do involve spatial land use changes, whereas the lumped models are useful for other types of scenarios studies and to reduce the computational times. For the lumped models, the calculation times were $10^{3}$ to $10^{6}$ shorter when compared to the detailed models. The lumped and spatial models moreover produce consistent results, as is shown by Tran Quoc and Willems (2016) for impact analysis of climate change scenarios.

\section{Discussion and conclusions}

The proposed approach successfully derived for the study catchment an accurate and fast combined conceptual rainfallrunoff and river hydraulic model. It was based on a modelling framework developed to incorporate several approaches to parsimoniously model the catchment hydrology and the hydraulics of rivers, floodplains and sewer systems. Albeit not shown in this paper, this flexible modelling framework can deliver hydrological and hydraulic models that can be configured with varying resolutions and provide consistent results. Further extensions are possible to the sewer systems in the catchment. The level of model detail can be tailored to the investigated scale, dynamics of the system and the intended application. Overly complicated models are both data- and time-demanding to set up. In addition, such complicatedness can lead to equifinality issues, prolonged calculation times, increased model uncertainties and even inaccurate simulations due to the accumulation of model uncertainties and discrepancies.

These models can easily be interfaced and employed for various water management studies. However, additional research remains necessary. Firstly, the incorporated modelling approaches require further developments. More model structures can be considered to identify and select the optimal one and/or to apply an ensemble approach to account for the model structure uncertainty. A sensitivity analysis may be performed to evaluate the parameters of the spatial aggregation and disaggregation steps. Also, the application of the models for scenario studies and water management decision making needs to be further studied. Both the hydraulic and hydrological modelling approach should be tested on different case studies. Secondly, future research can focus on the development of a decision support system for water management on catchment scale that incorporates the modelling framework. This requires further enhancement of the PCRaster-Python and CMD software tools to ensure that the hydrological and hydraulic conceptual models can be configured easily and quickly. Also, additional developments are necessary to (semi-automatically) interface the obtained models. 
Acknowledgements. The authors would like to thank DHI for the MIKE11 license. The Province of Antwerp and the Flemish Environment Agency (VMM) are also gratefully acknowledged for providing measurement data of the cross-sections of the rivers in the Grote Nete catchment such that the hydrodynamic river model could be set up.

\section{References}

Ajami, N. K., Gupta, H., Wagener, T., and Sorooshian, S.: Calibration of a semi-distributed hydrologic model for streamflow estimation along a river system, J. Hydrol., 298, 112-135, 2004.

Box, G. E. P. and Cox, D. R.: An analysis of transformations, J. Roy. Stat. Soc., 26, 211-243, 1964.

Breuer, L., Huisman, J. A., Willems, P., Bormann, H., Bronstert, A., Croke, B. F. W., Frede, H.-G., Gräff, T., Hubrechts, L., Jakeman, A. J., Kite, G., Lanini, J., Leavesley, G., Lettenmaier, D. P., Lindström, G., Seibert, J., Sivapalan, M., and Viney, N. R.: Assessing the impact of land use change on hydrology by ensemble modeling (LUCHEM) I: Model intercomparison with current land use, Adv. Water Resour., 32, 129-146, 2009.

De Vleeschauwer, K., Weustenraad, J., Nolf, C., Wolfs, V., De Meulder, B., Shannon, K., and Willems, P.: Green-blue water in the city: quantification of impact of source control versus end-ofpipe solutions on sewer and river floods, Water Sci. Technol., 70, 1825-1837, 2014.

Jakeman, A. J., Littlewood, I. G., and Whitehead, P. G.: Computation of the instantaneous unit hydrograph and identifiable component flows with application to two small upland catchments, J. Hydrol., 117, 275-300, 1990.

Keupers, I., Wolfs, V., Kroll, S., and Willems, P.: Impact analysis of sewer overflows on the receiving river water quality using an integrated conceptual model, Proceedings of the 10th conference on Urban Drainage Modelling (10UDM), 20-23 September 2015, Québec, Canada, 2015.

Moore, R. J.: The PDM rainfall-runoff model, Hydrol. Earth Syst. Sci., 11, 483-499, doi:10.5194/hess-11-483-2007, 2007.
Nielsen, S.-A. and Hansen, E.: Numerical simulation of the rainfallrunoff process on a daily basis, Nord. Hydrol., 4, 171-190, 1973.

Ravazi, S., Tolson, B. A., and Burn, D. H.: Review of surrogate modeling in water resources, Water Resour. Res., 48, W07401, doi:10.1029/2011WR011527, 2012.

Tran Quoc, Q. and Willems, P.: Flexible conceptual hydrological modelling - Disaggregation from lumped catchment scale to higher spatial resolutions, submitted, 2016.

Vansteenkiste, T., Tavakoli, M., Ntegeka, V., De Smedt, F., Batelaan, O., Pereira, F., and Willems, P.: Intercomparison of hydrological model structures and calibration approaches in climate scenario impact projections, J. Hydrol., 519, 743-755, 2014.

Vermuyten, E., Meert, P., Wolfs, V., and Willems, P.: Using a fast conceptual river model for floodplain inundation forecasting and real-time flood control - a case study in Flanders, Belgium. 21st International Congress on Modelling and Simulation (MODSIM2015), 29 November-4 December 2015, Broadbeach, Queensland, Australia, 2015.

Wheather, H. S., Jakeman, A. J., and Beven, K. J.: Progress and directions in rainfall-runoff modeling, in: Modelling change in environmental systems, edited by: Jakeman, A. J., Beck, M. B., and McAleer, M. J., John Wiley \& Sons, 102-132, 1993.

Willems, P.: A time series tool to support the multi-criteria performance evaluation of rainfall-runoff models, Environ. Model. Softw., 24, 311-321, 2009.

Willems, P.: Parsimonious rainfall-runoff model construction supported by time series processing and validation of hydrological extremes - Part 1: Step-wise model-structure identification and calibration approach, J. Hydrol., 510, 578-590, 2014.

Wolfs, V., Van Steenbergen, N., and Willems, P.: Flood probability mapping by means of conceptual modelling, in: River Flow 2012: Vol. 2. International Conference on Fluvial Hydraulics, edited by: Muñoz, R., Costa Rica, 5-7 September 2012, 10811085, London: CRC Press, Taylor \& Francis Group, 2012.

Wolfs, V., Meert, P., and Willems, P.: Modular conceptual modelling approach and software for river hydraulic simulations, Environ. Model. Softw., 71, 60-77, 2015. 